\title{
Adaptação de cônjuges e de expatriados e sua relação com o desempenho em designações internacionais
}

\author{
Brunella Tristão Simonelli ${ }^{\mathrm{A} 1}$ e Bruno Felix von Borell de Araujo ${ }^{\mathrm{B}}$ \\ ${ }^{A}$ Faculdade Pitágoras Linhares, Linhares, ES, Brasil \\ ${ }^{B}$ Fundação Instituto Capixaba de Pesquisas em Contabilidade, Economia e Finanças - FUCAPE, Vitória, ES, Brasil
}

\section{DETALHES DO ARTIGO}

\section{Histórico do artigo:}

Recebido em 22 de julho de 2015

Aceito em 1 de abril de 2016

Disponível online em 31 de agosto de 2016

Sistema de Revisão "Double Blind Review"

Editor científico:

Eduardo Eugênio Spers

\section{Palavras-chaves:}

Adaptação

Expatriados

Cônjuge

Desempenho

\begin{abstract}
RESUMO
A adaptação de um expatriado é função de diversos fatores, e questões familiares têm sido apontadas como possíveis influenciadores nesse processo. Entretanto, pouco se sabe sobre o efeito existente do papel do cônjuge na adaptação do expatriado. Assim, o objetivo deste trabalho é avaliar a relação entre a adaptação de expatriados e seus cônjuges com o desempenho do expatriado. Trata-se de um estudo quantitativo, realizado com 217 casais com um dos cônjuges tendo ocupado a posição de expatriado. Os resultados não mostraram uma relação significante entre a adaptação do cônjuge e o desempenho do expatriado, mas revelaram que a adaptação do expatriado modera a relação entre essas variáveis. Esses resultados sugerem que a adaptação do cônjuge pode exercer uma influência indireta no desempenho do expatriado, via sua adaptação. Esses achados suportam a ideia de que deve-se investir em suporte organizacional para facilitar um melhor ajuste do expatriado e seu cônjuge à cultura do país de destino e promover um desempenho individual superior.
\end{abstract}

(C) 2016 Internext | ESPM. Todos os direitos reservados!

\section{Introdução}

O comércio internacional exerce um importante papel na economia global e domina parte considerável das atividades do comércio mundial (Santos, Bronzo, Oliveira, \& Resende, 2014). Interações além das fronteiras nacionais têm permeado as atividades diárias de empresas de todo o mundo, impactando no aumento do número de expatriados (Kumar, Rose, \& Ramalu, 2008; Mohr \& Klein, 2004). Dadas as altas taxas de insucesso de gestores em missões internacionais, pesquisadores e gestores têm procurado desenvolver uma visão mais estratégica do sistema de pessoal global (Selmer \& Leung, 2003; Lazarova \& Cerdin, 2007) e melhor compreender fatores que facilitam ou dificultam o alcance dos resultados esperados em processos de expatriação (Takeuchi, Lepak, Marinova, \& Yun, 2007).
Neste contexto, alguns autores têm sugerido que uma das principais razões para a falta de adaptação do expatriado à designação internacional é justamente a dificuldade de ajuste do cônjuge (Andreason, 2008; Cole, 2011). Cerca de 80\% dos expatriados são acompanhados pelo cônjuge/parceiro durante suas missões internacionais (BGRS, 2014). Dessa forma, para reduzir os índices de insucesso nas designações, além de melhorar o desempenho do expatriado, sua adaptação se torna imprescindível, e muitas empresas têm investido em onerosos pacotes de benefícios destinados a expatriados e seus cônjuges, de forma a promover a adaptação de ambos (Bauer \& Taylor, 2001; Cole, 2011). Apesar de essa ser uma prática recorrente, pesquisas têm mostrado resultados divergentes no tocante a explicar até que ponto o a adaptação de expatriados pode ser influenciada pela adaptação do seu cônjuge (Davies,

\footnotetext{
${ }^{1}$ Contato do autor: Email: brunella@talentorh.net
} 
Kraeh, \& Froese, 2015; Lee \& Kartika, 2014; Van Erp, Van Der Zee, Giebels, \& Van Dujin, 2014).

De forma a preencher essa lacuna na literatura, o objetivo deste trabalho é avaliar a relação entre a adaptação de expatriados e seus cônjuges com o desempenho do expatriado. As contribuições deste estudo se dão pelo avanço no conhecimento da literatura sobre Gestão de Recursos Humanos Internacionais acerca das relações entre adaptação do cônjuge, adaptação do expatriado e desempenho do expatriado, de forma que esse resultado se some aos já realizado no sentido de melhor compreender as relações entre as variáveis estudadas. Em termos práticos, fornece subsídios para as decisões de investir em pacotes de suporte para expatriados e seus cônjuges.

\section{Desempenho de expatriados}

O desempenho de expatriados é um tema de extrema importância para as organizações, dado que o investimento feito em designações internacionais é de alto custo (McNulty, De Cieri, \& Hbutchings, 2009). Para as organizações, um desempenho satisfatório é entendido como o desenvolvimento bem sucedido das obrigações no exterior, incluindo o alcance dos objetivos específicos ou projetos definidos (BhaskarShrinivas, Harrison, Shaffer, \& Luk, 2005). Alguns expatriados cumprem suas designações de maneira satisfatória, dando seguimento à expatriação, mesmo sem estarem em condições adequadas para terem um desempenho satisfatório. Entretanto, mensurar esse desempenho não é uma tarefa simples.

A literatura apresenta, em várias perspectivas teóricas, explicações sobre desempenho em designações internacionais. Essas explicações, contudo, não se destinam a cobrir todas as dimensões possíveis, e sim a identificar as principais variáveis que predizem o desempenho dos expatriados, desde as características de personalidade, às questões de adaptação cultural. Nesse sentido, teóricos têm abordado características de personalidade, adaptação do expatriado e sua família, ajuste ao ambiente, estabelecimento de redes sociais, cultura, estrutura e governança organizacional (Reis, Fleury, Fleury \& Zambaldi, 2015; Claus, Lung, \& Bhattacharjee, 2011) como aspectos componentes do desempenho do expatriado.

Alguns autores propuseram estruturas de dimensões para a mensuração do desempenho. Por exemplo, Foster e Johnson (1996) sugeriram que três dimensões, a saber: adaptação transcultural, cumprimento da designação internacional e desempenho durante a designação (Caligiuri \& Tung, 2001; Schuler, Fulkerson, \& Downling, 1991). Claus, Lung, e Bhattacharjee (2011), por sua vez, destacam as dimensões de desempenho individual, laboral ou social. Caligiuri (2000) propôs cinco grandes traços de personalidade para a avaliação do desempenho. Já Kraimer e Wayne (2004) avaliam o desempenho na tarefa e a intenção de completá-la.

Assim como Cheng e Lin (2009), este estudo utilizou apenas a terceira dimensão (desempenho durante a designação) de Foster e Johnson (1996) como representativa do desempenho, pois se entende serem a adaptação e o cumprimento da designação construtos discriminantes possuidores de relação com o desempenho, apesar de não refletirem o seu conceito. Seguindo a adaptação feita por Cheng e Lin (2009) para a mensuração dos autores anteriormente citados, adotou-se nesta pesquisa o entendimento de que o desempenho do expatriado durante a designação (uma das três dimensões componentes do desempenho do expatriado), também é tridimensional e composto das seguintes dimensões:

- Eficiência da gestão, relacionada com o alcance das metas de produção com eficiência e qualidade;

- Gerenciamento dos empregados locais, o que diz respeito à supervisão, à implementação das políticas de $\mathrm{RH}$ e à redução das taxas de rotatividade;

- Leitura do ambiente, no que tange ao apoio à subsidiária por meio do estabelecimento de rede social com o governo local e demais pessoas influentes, da previsão de tendências regionais e da participação mais ativa nas associações do setor.

Essa abordagem possibilita entender o desempenho por meio da capacidade de o expatriado fazer uma leitura eficaz do ambiente, de forma a obter o melhor desempenho dos empregados locais, alcançando, assim, a eficiência da gestão em produção, qualidade e otimização de tempo e recursos. Neste estudo, delineia-se o interesse em avaliar a adaptação transcultural de expatriados como antecedente do seu desempenho, o que nos leva ao próximo tópico. 


\section{Adaptação Transcultural de Expatriados}

A adaptação transcultural de expatriados diz respeito à facilidade ou dificuldade enfrentada pelas pessoas em diversos aspectos da vida e do trabalho em designações internacionais (Lee \& Vorst, 2010). O expatriado bem ajustado se sente confortável em relação às suas tarefas e à interação com as pessoas do país que o acolhe, com reflexo na percepção de um maior desempenho por parte de seus gestores (Kraimer, Wayne, \& Jaworski, 2001). Ao longo do tempo, a diminuição das incertezas por meio do aprendizado de comportamentos apropriados à nova cultura traz maior harmonia à missão internacional (Black, 1988). Apesar de apontada frequentemente como componente do desempenho do expatriado (Schuler et al., 1991), apresentamos neste estudo a adaptação transcultural como um constructo distinto, com conteúdo substantivo próprio.

Em razão da crescente importância do construto "Adaptação Transcultural", o tema passou a ser objeto de iniciativas teóricas e empíricas que permitisse identificar suas dimensões. Black (1988) elaborou um modelo apresentando três facetas da adaptação transcultural de expatriados: a adaptação geral (envolvendo clima, alimentação e habitação), a adaptação ao trabalho (envolvendo expectativas e valores relativos às próprias tarefas laborais) e, finalmente, a adaptação à interação (envolvendo as relações interpessoais). Essas três facetas não ocorrem de forma isolada. Ao invés disso, influenciam-se mutuamente. Sendo assim, a falta de domínio do idioma pode minimizar o sucesso na interação social, bem como o desenvolvimento de algumas atividades no próprio trabalho, nas compras, no deslocamento e, por conseguinte, prejudicam a adaptação geral (Selmer \& Fenner Jr., 2008).

A adaptação transcultural é apontada na literatura com uma pré-condição teórica para o sucesso de designações internacionais. Dificuldades na adaptação à cultura em designações internacionais trazem ao expatriado um desempenho abaixo do esperado (Pattie \& Parks, 2011; Shih, Chiang, \& Hsu, 2010), além de poder encerrar prematuramente a designação ou culminar no próprio desligamento (Caligiuri \&Tung, 2001; Ramalu, Wei, \& Rose, 2011; Shaffer, Harrison, Gregersen, Black, \& Ferzandi, 2006). Como as expatriações têm se tornado uma vantajosa opção para o desenvolvimento de habilidades globais (Takeuchi et al., 2007) e, consequentemente, criam vantagem competitiva para a organização (Collings, Scullion, \& Morley, 2007; Shih et al., 2010), pesquisadores e gestores devem ter uma visão mais estratégica do sistema de pessoal global (Lazarova \& Cerdin, 2007; Selmer \& Leung, 2003), principalmente porque as expatriações tornaram-se uma etapa necessária para o gerenciamento de carreiras executivas (Takeuchi et al., 2007).

É possível também encontrar na literatura evidências empíricas para a relação positiva entre adaptação do expatriado e seu desempenho (Kraimer et al., 2001; Kumar et al., 2008; Shih et al., 2010; Wu \& Ang, 2011). Kraimer, Wayne, e Jaworski (2001) identificaram que o fracasso do expatriado em suas atividades laborais é, muitas vezes, resultado da má adaptação. Kumar, Rose, \& Ramalu (2008) argumentam que a adaptação transcultural do expatriado reduz o estresse e a tensão da designação, o que contribui para melhorar o desempenho, já que ajuda o expatriado a economizar energia e esforços em seu trabalho.

Assim, diante de tais evidências, espera-se que os indivíduos com melhor ajuste geral e de interação sejam capazes de executar níveis mais elevados em suas atividades laborais. Da relação entre a adaptação do expatriado e seu desempenho, surge a primeira hipótese do presente trabalho.

\section{H1: $O$ desempenho do expatriado está positivamente relacionado com a adaptação do expatriado.}

\section{Adaptação Transcultural de Cônjuges/parceiros de expatriados}

Se a adaptação de expatriados vem sem do explorada de forma ampla na literatura, o mesmo não se pode dizer em relação ao processo de ajuste que ocorre com maridos e esposas. A adaptação do cônjuge não é bem compreendida, e o que se sabe sobre a adaptação do expatriado não é suficiente para entender as dificuldades com as quais os cônjuges se deparam (Andreason, 2008; Bauer \& Taylor, 2001). Os estudos sobre adaptação do cônjuge tendem mais a avaliar empiricamente os fatores envolvidos nessa adaptação do que a fornecer uma definição propriamente dita (Black \& Gregersen, 1991; Black \& Stephens, 1989; Takeuchi et al., 2007; Van Der Zee, Ali, \& Salomé, 2005).

$\mathrm{Na}$ literatura, notam-se diferentes perspectivas para o entendimento a respeito do que constitui 
adaptação do cônjuge/parceiro. Shaffer e Harrison (1998), por exemplo, concluíram ser a adaptação do cônjuge constituída de três dimensões: a forma como o cônjuge se ajusta aos costumes e cultura local; como ele constrói relações com os cidadãos locais e o sentido dado por ele ao fato de fazer parte daquela cultura, sentindo-se em casa. Diante disso, o ajustamento nessas três dimensões depende do estabelecimento de sua identidade na nova cultura (Bauer \& Taylor, 2001).

Por sua vez, Mohr e Klein (2004) identificaram três dimensões do ajuste de cônjuges, em estudo com cônjuges americanos na Alemanha. Neste aspecto, duas das dimensões sugeridas por Black e Gregersen (1991) foram confirmadas (adaptação geral e adaptação à interação), mas uma terceira dimensão foi acrescida: a adaptação ao papel de cônjuge de expatriado. Mohr e Klein (2004) verificam estarem a adaptação geral e a adaptação ao papel associadas positivamente com experiências internacionais anteriores, embora tais experiências tenham tido uma relação negativa com a adaptação à interação. Isso pode ser explicado pelas diferenças culturais entre o país anteriormente visitado e o de moradia atual. Essa pesquisa, porém, objetivou conhecer os antecedentes da adaptação do cônjuge (Mohr \& Klein, 2004). Este estudo baseia-se nesses autores para operacionalizar a adaptação do cônjuge.

É importante ressaltar que embora muitas pessoas pensem no papel de esposa, ao se referirem ao termo "cônjuge de expatriado", nem sempre este é o caso. Apesar de, em sua maioria, os expatriados serem do sexo masculino, a presença das mulheres em designações internacionais é cada vez maior (Andreason, 2008). Atualmente, 18\% dos expatriados são mulheres (BGRS, 2014), que assumem suas atribuições acompanhadas de cônjuges os quais deixam uma carreira no país de origem e precisam lidar com a possibilidade da não recolocação no país de destino, por não dominarem o idioma, ou devido ao caráter temporário da designação (Andreason, 2008). De forma geral, a adaptação para cônjuge do sexo feminino pode ser menos difícil do que para o do masculino. Fora isso, as dificuldades para recolocação na cultura de destino dos cônjuges que têm carreira (dual couple career) são maiores, e isso reflete em uma proporcional dificuldade de adaptação, se comparada à dos cônjuges sem carreira antes da transferência internacional (Cole, 2011; Harvey, 1998; Harvey, Napier, \& Moeller, 2009), por terem mais obstáculos a superar, na tentativa de criar nova rede profissional e um sistema de apoio, autonomamente, enquanto seu parceiro passa longas horas no trabalho e viaja com frequência (Kupka \& Cathro, 2007; Shaffer \& Harrison, 2001). Mesmo quando conseguem um emprego no exterior, não têm benefícios como os fornecidos ao expatriado organizacional, como treinamento sobre a cultura local e o idioma (Andreason, 2008; Anderson, Ones, Sinangil, \& Viswesvaran, 2001). Tais fatores talvez justifiquem o fato de famílias de dupla carreira serem mais resistentes à realização de trabalhos no exterior (Harvey et al., 2009).

Não têm sido raras as evidências empíricas para a relação positiva entre a adaptação do cônjuge e a do expatriado (por exemplo, Andreason, 2008; Bauer \&Taylor, 2001; Black \& Stephens, 1989; Brown, 2008; Harvey et al., 2009; Konopaske, Robie, \& Ivancevich, 2005; Kupka \& Cathro, 2007), embora haja também os que apresentam resultados divergentes (Davies et al., 2015; Lee \& Kartika, 2014; Van Erp et al., 2014). Em geral, esses estudos sugerem que o suporte oferecido pelo cônjuge em termos emocionais, de organização da vida familiar, aconselhamento e sincronização de interesses com as redes sociais locais e do país de origem tendem a apoiar o ajuste do expatriado ao país de destino na designação internacional, assim como seu desempenho profissional.

Diante disto, apresentam-se as seguintes hipóteses:

\section{H2: Adaptação do cônjuge está positivamente relacionada com a adaptação do expatriado.}

\section{H3: Adaptação do cônjuge está positivamente relacionada com 0 desempenho do expatriado.}

\section{Metodologia}

\subsection{Coleta de dados}

Os sujeitos da pesquisa são casais formados por um expatriado e cônjuge/companheiro, que o acompanhou durante a designação para trabalhar no Brasil. Os dois questionários estruturados aplicados (um com questões destinadas ao expatriado e outro com questões destinadas ao seu cônjuge/companheiro) foram hospedados em um website. Os links para acesso aos questionários foram encaminhados em um e-mail para um grupo de 523 expatriados organizacionais (EO) em designações no 
Brasil. Para as respostas obtidas serem provenientes de expatriados e seus cônjuges, foi esclarecido no $e$ mail convite ser o interesse da pesquisa a participação de expatriados casados ou que tivessem um (a) companheiro (a).

Por meio de blogs, redes sociais sobre expatriados no Brasil e contatos com o departamento de Recursos Humanos de 53 empresas que recebiam estrangeiros em designações internacionais no Brasil, foi criada uma lista de e-mails, ao longo de 12 meses, para os quais o link da pesquisa foi enviado. Após serem encontrados os primeiros expatriados por essas fontes, adotou-se a técnica de amostragem bola de neve (Heckathorn, 1997), e foi solicitado aos expatriados indicarem outros participantes os quais compusessem a amostra almejada, o que pode aumentar a composição da base de e-mails usada para a coleta de dados neste estudo. Essa estratégia vem sendo utilizada em pesquisas recentes com expatriados (p.e., Araujo, Teixeira, Cruz, \& Malini, 2014).

A coleta de dados se deu ao longo de 18 dias, em maio de 2012, sendo enviado um comunicado intermediário no 13으 dia. Dos 226 retornos recebidos, 9 foram eliminados devido à repetição das respostas, critério apontado pelo ESS-EDUNET European Social Survey Education Net (2009) que recomenda como aceitável um percentual de dados faltantes de $23,8 \%$ e de $76,2 \%$ para repetição de respostas. Dos questionários, 217 foram considerados válidos e sem missing values, refletindo uma taxa de retorno de 41,50\%. Pela regra empírica de cinco a dez casos por variável (Hair Jr., Anderson, Tatham, \& Black, 2005), a amostra de 217 casais pode ser considerada de tamanho adequado para os fins do estudo, por ser o instrumento de coleta de dados composto de 29 assertivas.

\subsection{Medidas}

A escala de Black (1988) foi o instrumento de avaliação do grau de adaptação transcultural dos expatriados nos três aspectos contemplados: a adaptação geral, a adaptação ao trabalho e, finalmente, a adaptação à interação. Essa mesma escala foi utilizada para a avaliação da adaptação transcultural do cônjuge/companheiro do expatriado, sem, entretanto, a terceira dimensão (adaptação ao trabalho) ser contemplada, totalizando 8 itens para os cônjuges e 11 para os expatriados. A exclusão dessa dimensão para os cônjuges se deu pelo fato de muitos deles não darem seguimento a uma carreira no país de acolhimento, tampouco estarem vinculados a uma organização. Assim, os cônjuges dos expatriados somente responderam às assertivas da escala de Black (1988) relativas às dimensões de adaptação geral e quanto à interação.

O instrumento foi apresentado em uma escala Likert de 5 (cinco) pontos, em que "1" (um) representa "não adaptado" e "5" (cinco) significa "muito bem adaptado". A escala foi aplicada em sua versão original no idioma inglês, dominado pelos expatriados, de nacionalidades diversas.

As informações sobre o desempenho dos executivos, independente de serem expatriados ou não, são de difícil acesso, uma vez que as organizações dificultam o fornecimento de tais dados, tratando-os com maior confidencialidade. Por esse motivo, o desempenho tratado no presente trabalho foi o percebido pelo próprio expatriado e não o avaliado pelos líderes. Tal estratégia tem sido adotada em estudos semelhantes os quais avaliam o desempenho dos expatriados (Hemmasi, Downes, \& Varner, 2010; Lee \& Sukoco, 2010).

Para mensurar o desempenho de expatriados, utilizou-se a escala de Cheng e Lin (2009), segundo a qual o constructo se apresenta de forma tridimensional e é entendido como a capacidade de o expatriado fazer uma leitura eficaz do ambiente, obtendo o melhor desempenho dos empregados locais, alcançando, assim, a eficiência da gestão em produção, qualidade e otimização de tempo e recursos. As três dimensões do desempenho avaliadas nos 10 itens da escala foram: "Eficiência da Gestão", "Gerenciamento dos Empregados Locais" e "Leitura do Ambiente". As respostas foram dispostas em escala Likert de 5 (cinco) pontos, na qual 1 (um) representa "discordo totalmente", e 5 (cinco) "concordo totalmente". A escala também foi aplicada no idioma inglês. As escalas poderão ser obtidas com os autores por correspondência direta.

As escalas foram escolhidas por terem suas definições operacionais alinhadas com as definições constitutivas apresentadas no referencial teórico e por suas dimensões apresentarem Alfas de Cronbach acima de 0,80 em suas aplicações originais (Black, 1988; Cheng \& Lin, 2009). 
5.3 Análise preliminar dos dados

Após a verificação dos dados faltantes (missing values) e atípicos (outliers), primeira etapa da análise, nenhum respondente foi eliminado. As respostas dos itens que utilizavam escala reversa foram recodificadas. As relações hipotetizadas foram analisadas mediante modelagem de equações estruturais (structural equation modeling - SEM), com estimação por mínimos quadrados parciais e do software SmartPLS 2.0M3 (Ringle, Wende, \& Will, 2005). O método PLS foi escolhido pelo fato de os dados não serem normais. Em seguida, avaliou-se o modelo de mensuração (validade convergente, validade discriminante e confiabilidade), além de ser efetuada a avaliação do modelo estrutural.

\section{Análise e discussão dos resultados}

\subsection{Dados demográficos}

A Tabela 1 mostra que a maioria dos expatriados participantes é do sexo masculino (89\%), casada legalmente (66\%), trabalha em filiais brasileiras de empresas de capital estrangeiro (69\%), ocupa posição gerencial (94\%) e está em sua primeira designação internacional (70\%). Um total de $65 \%$ dos casais participantes possui 1 ou 2 filhos. Esses resultados se

Tab. 1

Dados Demográficos

\begin{tabular}{|c|c|c|c|c|c|c|}
\hline \multicolumn{2}{|c|}{ Perfil dos participantes } & \multirow{2}{*}{$\begin{array}{c}\text { no } \\
193 \\
\end{array}$} & \multirow{2}{*}{$\begin{array}{l}\% \\
89 \\
\end{array}$} & \multirow{2}{*}{$\begin{array}{l}\text { País de origem } \\
\text { dos expatriados } \\
\text { Estados Unidos }\end{array}$} & \multirow{2}{*}{$\begin{array}{l}\text { no } \\
43 \\
\end{array}$} & \multirow{2}{*}{$\begin{array}{c}\% \\
19,8\end{array}$} \\
\hline Gênero do & Masculino & & & & & \\
\hline Expatriado & Feminino & 24 & 11 & Argentina & 19 & 8,8 \\
\hline \multirow{5}{*}{$\begin{array}{c}\text { Faixa etária } \\
\text { do expatriado }\end{array}$} & Até 35 anos & 33 & 15 & Portugal & 17 & 7,8 \\
\hline & Entre 36 e 40 anos & 40 & 18 & China & 14 & 6,5 \\
\hline & Entre 41 e 45 anos & 58 & 27 & México & 14 & 6,5 \\
\hline & Entre 46 e 50 anos & 51 & 24 & Espanha & 12 & 5,5 \\
\hline & A partir de 51 anos & 35 & 16 & Inglaterra & 10 & 4,6 \\
\hline \multirow{5}{*}{$\begin{array}{l}\text { Faixa etária } \\
\text { do cônjuge }\end{array}$} & Até 35 anos & 44 & 20 & Japão & 10 & 4,6 \\
\hline & Entre 36 e 40 anos & 60 & 28 & Colômbia & 8 & 3,7 \\
\hline & Entre 41 e 45 anos & 55 & 25 & Peru & 8 & 3,7 \\
\hline & Entre 46 a 50 anos & 39 & 18 & França & 7 & 3,2 \\
\hline & A partir de 51 anos & 19 & 9 & Itália & 7 & 3,2 \\
\hline \multirow[t]{4}{*}{ Tempo no Brasil } & Entre 12 e 24 meses & 55 & 25 & Venezuela & 7 & 3,2 \\
\hline & Entre 25 e 36 meses & 58 & 27 & Alemanha & 6 & 2,8 \\
\hline & Entre 37 e 48 meses & 91 & 42 & Noruega & 6 & 2,8 \\
\hline & A partir de 49 meses & 13 & 6 & Uruguai & 5 & 2,3 \\
\hline \multirow[t]{2}{*}{ Estado civil } & Casados & 143 & 66 & Canadá & 4 & 1,8 \\
\hline & Morando com o parceiro & 74 & 34 & Irlanda & 4 & 1,8 \\
\hline \multirow{5}{*}{$\begin{array}{l}\text { Tempo de vida } \\
\text { em casal }\end{array}$} & Até 5 anos & 29 & 13 & Suécia & 4 & 1,8 \\
\hline & De 6 a 10 anos & 48 & 22 & Finlândia & 3 & 1,4 \\
\hline & De 11 a 15 anos & 54 & 25 & Holanda & 3 & 1,4 \\
\hline & De 16 a 20 anos & 51 & 24 & Dinamarca & 2 & 0,9 \\
\hline & Acima de 20 anos & 35 & 16 & Austrália & 1 & 0,5 \\
\hline \multirow[t]{4}{*}{ Número de filhos } & Nenhum & 53 & 24 & Bélgica & 1 & 0,5 \\
\hline & 1 filho & 82 & 38 & Equador & 1 & 0,5 \\
\hline & 2 filhos & 58 & 27 & Nova Zelândia & 1 & 0,5 \\
\hline & 3 filhos ou mais & 24 & 11 & & & \\
\hline \multirow{2}{*}{$\begin{array}{l}\text { Nacionalidade da } \\
\text { organização }\end{array}$} & Brasileira & 68 & 31 & & & \\
\hline & Estrangeira & 149 & 69 & & & \\
\hline \multirow[t]{2}{*}{ Nível hierárquico } & Posição Gerencial & 203 & 94 & & & \\
\hline & Não Gerencial & 14 & 6 & & & \\
\hline \multirow{2}{*}{$\begin{array}{c}\text { Experiência prévia } \\
\text { em expatriação }\end{array}$} & Sim & 65 & 30 & & & \\
\hline & Não & 152 & 70 & & & \\
\hline
\end{tabular}

Fonte: Dados da Pesquisa 
mostram coerentes com o perfil demográfico global de expatriados identificado pelo BGRS (2014).

As nacionalidades mais presentes dentre os expatriados participantes são as de estadunidenses $(19,8 \%)$, argentinos $(8,8 \%)$, portugueses $(7,8 \%)$, chineses $(6,5 \%)$ e mexicanos (6,5\%). Com exceção de apenas um expatriado norueguês e outro espanhol, que são casados com brasileiras, todos os cônjuges participantes possuem a mesma nacionalidade dos expatriados de quem são companheiros.

\subsection{Avaliação do Modelo de Mensuração}

Realizou-se a análise do modelo de mensuração antes da verificação das relações entre os constructos. As validades convergente e discriminante e a confiabilidade composta foram analisadas. A existência da validade convergente foi indicada com a observação de todas as variáveis latentes, de primeira e de segunda ordem, que apresentaram variância média extraída (average variance extracted - AVE) superior a 50\%, atendendo aos critérios propostos por Chin (1998) e Hair Jr., Anderson, Tatham, e Black, (2005), conforme apresentado nas Tabelas 3 e 4. Já a confiabilidade composta superior a 0,6 indica a confiabilidade do constructo em estudos que utilizam equações estruturais (Chin, 1998). Como ilustrado nas Tabelas 3 e 4 , nesta pesquisa todas as variáveis latentes apresentaram confiabilidade superior a 0,6, indicando a confiabilidade do modelo de mensuração.

A validade discriminante também foi confirmada no modelo de mensuração, em virtude de todos os indicadores possuírem cargas mais altas em seus constructos do que em qualquer outra variável

Tab. 2

Matriz de Cargas Cruzadas

\begin{tabular}{|c|c|c|c|c|c|c|c|c|c|}
\hline VL 10 ordem & Indicadores & 1 & 2 & 3 & 4 & 5 & 6 & 7 & 8 \\
\hline \multirow[t]{6}{*}{ 1. Adaptação geral (expatriado) } & AGE_1) & 0,74 & 0,69 & 0,31 & 0,18 & 0,26 & 0,47 & 0,54 & 0,57 \\
\hline & AGE_2) & 0,80 & 0,73 & 0,17 & 0,08 & 0,15 & 0,55 & 0,59 & 0,62 \\
\hline & AGE_3) & 0,85 & 0,81 & 0,22 & 0,12 & 0,22 & 0,54 & 0,63 & 0,64 \\
\hline & AGE_4) & 0,77 & 0,71 & 0,30 & 0,20 & 0,29 & 0,51 & 0,57 & 0,60 \\
\hline & AGE_5) & 0,70 & 0,68 & 0,41 & 0,35 & 0,35 & 0,55 & 0,57 & 0,64 \\
\hline & AGE_6) & 0,92 & 0,83 & 0,51 & 0,25 & 0,33 & 0,64 & 0,72 & 0,77 \\
\hline \multirow[t]{2}{*}{ 2. Adaptação à interação (expatriado) } & AIE_1) & 0,88 & 0,93 & 0,37 & 0,29 & 0,32 & 0,59 & 0,68 & 0,71 \\
\hline & AIE_2) & 0,84 & 0,92 & 0,36 & 0,27 & 0,35 & 0,55 & 0,69 & 0,71 \\
\hline \multirow[t]{3}{*}{ 3. Adaptação ao Trabalho (expatriado) } & ATE_1) & 0,32 & 0,33 & 0,81 & 0,63 & 0,59 & 0,40 & 0,44 & 0,47 \\
\hline & ATE_2) & 0,23 & 0,23 & 0,80 & 0,65 & 0,51 & 0,36 & 0,42 & 0,41 \\
\hline & ATE_3) & 0,37 & 0,33 & 0,70 & 0,27 & 0,21 & 0,33 & 0,38 & 0,45 \\
\hline \multirow[t]{6}{*}{ 4. Adaptação geral (cônjuge) } & AGC_1) & 0,30 & 0,33 & 0,55 & 0,82 & 0,65 & 0,29 & 0,35 & 0,34 \\
\hline & AGC_2) & 0,18 & 0,24 & 0,54 & 0,88 & 0,73 & 0,26 & 0,24 & 0,27 \\
\hline & AGC_3) & 0,22 & 0,25 & 0,64 & 0,85 & 0,61 & 0,27 & 0,36 & 0,35 \\
\hline & AGC_4) & 0,22 & 0,27 & 0,56 & 0,90 & 0,70 & 0,30 & 0,31 & 0,33 \\
\hline & AGC_5) & 0,20 & 0,25 & 0,54 & 0,85 & 0,65 & 0,24 & 0,27 & 0,27 \\
\hline & AGC_6) & 0,17 & 0,21 & 0,59 & 0,84 & 0,65 & 0,21 & 0,28 & 0,25 \\
\hline \multirow[t]{2}{*}{ 5. Adaptação à Interação (cônjuge) } & AIC_1) & 0,31 & 0,34 & 0,50 & 0,63 & 0,89 & 0,27 & 0,33 & 0,34 \\
\hline & AIC_2) & 0,30 & 0,32 & 0,52 & 0,76 & 0,92 & 0,28 & 0,32 & 0,37 \\
\hline \multirow[t]{3}{*}{ 6. Desempenho Gerencial } & DG_1) & 0,49 & 0,45 & 0,35 & 0,24 & 0,24 & 0,70 & 0,55 & 0,54 \\
\hline & DG_2) & 0,44 & 0,42 & 0,32 & 0,16 & 0,11 & 0,65 & 0,52 & 0,42 \\
\hline & DP_3) & 0,51 & 0,44 & 0,33 & 0,24 & 0,29 & 0,76 & 0,59 & 0,59 \\
\hline \multirow[t]{4}{*}{ 7. Desempenho de Leitura do Ambiente } & DLA_1) & 0,51 & 0,52 & 0,44 & 0,31 & 0,27 & 0,51 & 0,72 & 0,60 \\
\hline & DLA_2) & 0,59 & 0,57 & 0,39 & 0,25 & 0,28 & 0,68 & 0,77 & 0,65 \\
\hline & DLA_3) & 0,57 & 0,54 & 0,35 & 0,25 & 0,22 & 0,52 & 0,70 & 0,57 \\
\hline & DLA_4) & 0,52 & 0,53 & 0,38 & 0,21 & 0,29 & 0,53 & 0,70 & 0,59 \\
\hline \multirow[t]{3}{*}{ 8. Desempenho de Produção } & DP_1) & 0,60 & 0,60 & 0,49 & 0,29 & 0,31 & 0,51 & 0,60 & 0,74 \\
\hline & DP_2) & 0,69 & 0,65 & 0,48 & 0,26 & 0,30 & 0,61 & 0,71 & 0,82 \\
\hline & DP_3) & 0,60 & 0,55 & 0,40 & 0,28 & 0,33 & 0,60 & 0,65 & 0,79 \\
\hline
\end{tabular}

Fonte: Dados da Pesquisa 
Tab. 3

Correlação de Pearson e Estatísticas Descritivas das Variáveis Latentes de 1o Ordem

\begin{tabular}{|c|c|c|c|c|c|c|c|c|}
\hline Variável Latente de $1^{\mathrm{a}}$ ordem & 1 & 2 & 3 & 4 & 5 & 6 & 7 & 8 \\
\hline 1. Adaptação Geral do Expatriado & 1,00 & & & & & & & \\
\hline 2. Adaptação quanto à Interação do Expatriado & 0,93 & 1,00 & & & & & & \\
\hline 3. Adaptação quanto Trabalho do Expatriado & 0,41 & 0,39 & 1,00 & & & & & \\
\hline 4. Adaptação Geral do Cônjuge & 0,25 & 0,30 & 0,67 & 1,00 & & & & \\
\hline 5. Adaptação quanto à Interação do Cônjuge & 0,34 & 0,37 & 0,57 & 0,78 & 1,00 & & & \\
\hline 6. Desempenho Geral do Expatriado & 0,68 & 0,62 & 0,47 & 0,30 & 0,31 & 1,00 & & \\
\hline 7. Desempenho de Leitura do Ambiente & 0,76 & 0,75 & 0,54 & 0,35 & 0,36 & 0,78 & 1,00 & \\
\hline 8. Desempenho de Produção & 0,81 & 0,77 & 0,58 & 0,35 & 0,40 & 0,73 & 0,83 & 1,00 \\
\hline Média & 2,69 & 2,62 & 2,73 & 2,78 & 3,03 & 3,61 & 3,18 & 2,71 \\
\hline Mediana & 3,00 & 3,00 & 3,00 & 3,00 & 3,00 & 4,00 & 3,00 & 3,00 \\
\hline Desvio padrão & 0,96 & 0,82 & 0,92 & 1,19 & 1,26 & 0,83 & 0,78 & 0,77 \\
\hline Variância média extraída & 0,64 & 0,85 & 0,59 & 0,74 & 0,81 & 0,50 & 0,52 & 0,61 \\
\hline Confiabilidade composta & 0,91 & 0,92 & 0,81 & 0,94 & 0,90 & 0,75 & 0,81 & 0,83 \\
\hline
\end{tabular}

Fonte: Dados da Pesquisa

latente (ver Tabela 2). Uma vez que modelos PLS não possuem índices de Goodness of fit, a qualidade do modelo estrutural proposto foi avaliada por meio do $\mathrm{R}^{2}$ das variáveis dependentes e o Stone-Geisser $\mathrm{Q}^{2}$ para testar a relevância preditiva (Chin, 2010). Dado que os valores foram estáveis para as distâncias de omissão, e a maioria do $Q^{2}$ foi positiva, entendeu-se que o modelo apresenta uma estabilidade adequada.

\subsection{Avaliação do Modelo Estrutural}

O modelo estrutural relaciona a variável "Adaptação do Cônjuge" com o constructo "Adaptação do Expatriado" e este com o "Desempenho do Expatriado", o qual, segundo o modelo, também é relacionado diretamente com a "Adaptação do Cônjuge". Em outras palavras, testa-se se o "Desempenho do Expatriado" é influenciado

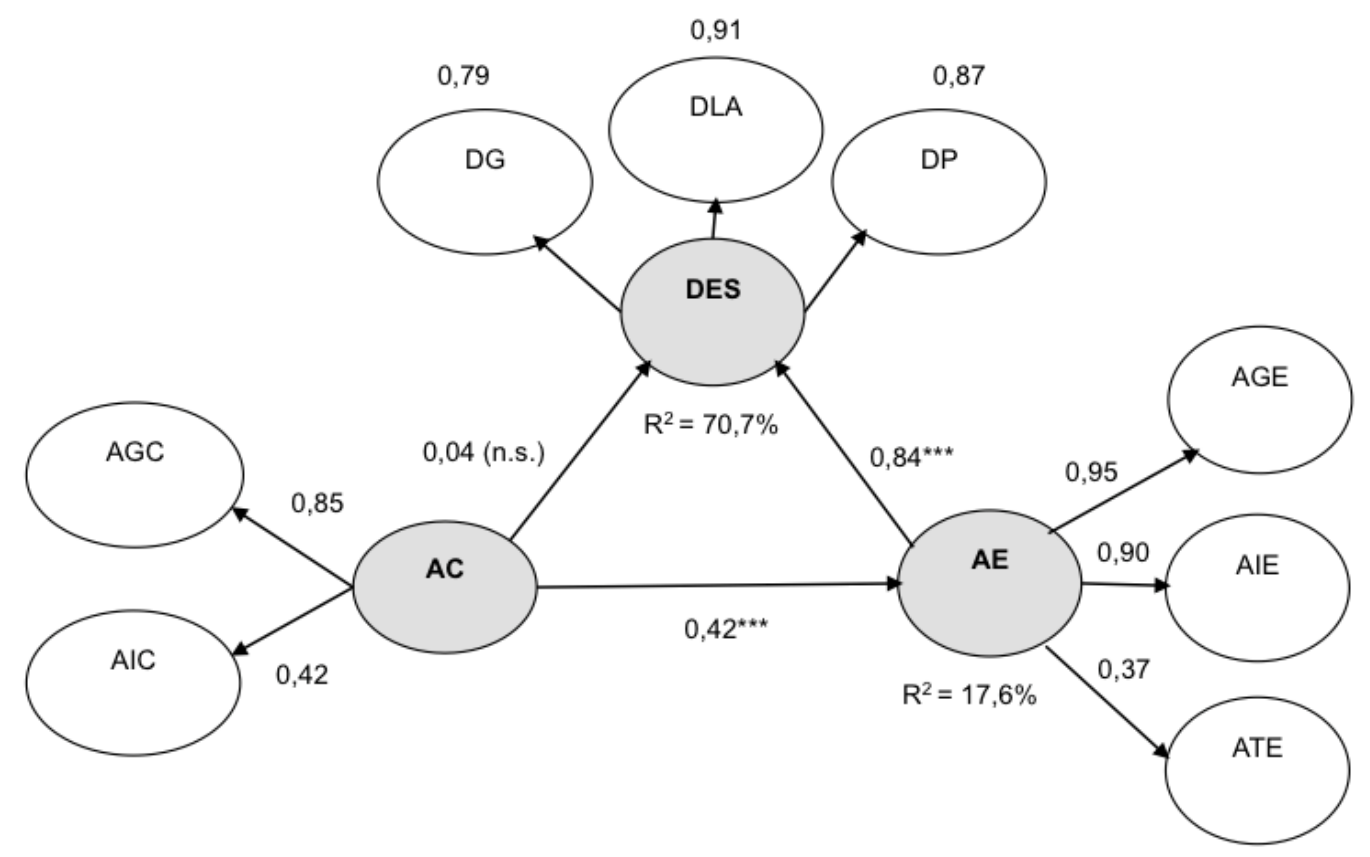

Fig. 1

Avaliação do Modelo Estrutural

Fonte: Dados da Pesquisa. Nota: Foi utilizado o software SmartPLS 2.0.M3 (Ringle et al., 2005). (n.s.) = Não Significante. *** Correlação é significante ao nível de 0,001. A significância foi estimada com N = 217 e 1.000 repetições, por meio de bootstrap. Para simplificar a figura, foram omitidos os indicadores, mas suas cargas fatoriais podem ser localizadas na Tabela 2. AGE = Adaptação Geral do Expatriado; AIE = Adaptação quanto à Interação do Expatriado; ATE = Adaptação quanto ao Trabalho do Expatriado AGC = Adaptação Geral do Cônjuge; AIC = Adaptação quanto à Interação do Cônjuge; DG = Desempenho Geral; DLA = Desempenho de Leitura do Ambiente; $D P=$ Desempenho de Produção. $A$ No modelo estrutural $A C=$ Adaptação do Cônjuge; $A E=$ Adaptação do Expatriado; DES = Desempenho do Expatriado. 
Tab. 4

Correlação de Pearson e Estatísticas Descritivas das Variáveis Latentes de 2a Ordem - Modelo Estrutural

\begin{tabular}{|c|c|c|c|}
\hline $\begin{array}{c}\text { Variáveis Latentes de } 20 \text { ordem } \\
\text { Modelo Estrutural }\end{array}$ & 1 & 2 & 3 \\
\hline 1. Adaptação do Cônjuge & 1 & 0 & 0 \\
\hline 2. Adaptação do Expatriado & 0,4191 & 1 & 0 \\
\hline 3. Desempenho do Expatriado & 0,389 & 0,8523 & 1 \\
\hline Média & 2,84735 & 2,688731 & 3,166359 \\
\hline Mediana & 3 & 3 & 3 \\
\hline Desvio padrão & 1,211032 & 0,928213 & 0,863411 \\
\hline Variância média extraída & 0,879103 & 0,72272 & 0,8531 \\
\hline Confiabilidade composta & 0,93552 & 0,94904 & 0,979687 \\
\hline
\end{tabular}

Fonte: Dados da Pesquisa

diretamente pela "Adaptação do Cônjuge" e indiretamente, por meio da "Adaptação do Expatriado". Realizada a análise proposta, obtiveramse os coeficientes de caminho apresentados na Figura 1.

O modelo estrutural foi estimado com o esquema de ponderação "path" e com base no modelo representado na Figura 1. É importante ressaltar que, consoante Cohen (1997), um $\mathrm{R}^{2}$ de $13 \%$ é classificado como médio, e um de 26\%, como grande. Dessa forma, de acordo com esse critério, o modelo apresentado apresenta uma explicação forte para o constructo desempenho do expatriado $\left(R^{2}=70,7 \%\right)$ e moderada para o constructo de adaptação do expatriado $\left(R^{2}=17,6 \%\right)$.

Os resultados mostraram uma relação positiva e significante entre o desempenho e a adaptação do expatriado (coeficiente de caminho $=0,84, p<0,001$ ), o que apoia a hipótese 1 (o desempenho do expatriado está positivamente relacionado com a adaptação do expatriado). Este resultado encontra suporte em estudos anteriores que apontam uma relação positiva entre esses constructos em diferentes contextos de pesquisa (Araujo, Teixeira, Cruz, \& Malini, 2012; Kraimer et al., 2001; Kumar et al., 2008; Shih et al., 2010; Wu \& Ang, 2011), o que também ocorre em outros estudos mais recentes (Davies et al., 2015; Lee \& Kartika, 2014; Van Erp et al., 2014). Somados, esses estudos parecem posicionar a adaptação transcultural do expatriado como uma variável de forte explicação sobre seu desempenho, o que ressalta a importância do constructo para a pesquisa em gestão internacional de Recursos Humanos.

A hipótese 2 (a adaptação do cônjuge está positivamente associada à adaptação do expatriado) também foi suportada, dado que foi encontrada uma relação positiva e significante entre os constructos apontados (coeficiente de caminhos $=0,42, p<0,001$ ). A associação entre a adaptação do cônjuge e a do expatriado já vinha sendo apontada na literatura em estudos anteriores (Andreason, 2008; Bauer \& Taylor, 2001; Kupka \& Cathro, 2007; Lee \& 2007; Shaffer \& Harrison, 1998; Takeuchi, Yun, \& Tesluk, 2002) e este estudo vem a somar-se àqueles no sentido de trazer novas evidências para essa associação. Brown (1997) identificou o fato de os casais apresentarem um stress semelhante, em virtude do reduzido tempo disponível para estarem juntos e das incertezas futuras, após a missão internacional. Outro dado desse estudo é que a dificuldade em conciliar as demandas familiares e profissionais se constitui como o principal fator estressor para os expatriados. Além disso, o entendimento da associação encontrada recebe contribuição dos efeitos de transbordamento (spillover effects) entre a adaptação de ambos (Andreason, 2008).

Por sua vez, a relação direta entre a adaptação do cônjuge e o desempenho do expatriado não encontrou suporte (coeficiente de caminho = 0,04, n.s.), e isso levou à rejeição de H3. Esse achado é particularmente interessante para a literatura sobre Gestão Internacional de Recursos Humanos, pois havia ainda uma necessidade de se testar essa relação direta, de forma a se avaliar como o ajuste do cônjuge pode apoiar o alcance de resultados por parte do expatriado. Esta pesquisa apresenta indícios de que o simples fato de se oferecer o suporte ao cônjuge não gera, por si, uma associação direta com o desempenho do executivo em uma designação internacional. Esse efeito tem caráter moderador, ou seja, se faz presente via adaptação dos expatriados. 
Em outras palavras, a adaptação do cônjuge do expatriado parece trazer consequências positivas para o desempenho se esse estado contribuir também para o ajuste do executivo, o que não é uma regra. Esse resultado fundamenta em termos empíricos essa ideia, que já vinha sendo ressaltada em estudos anteriores (Van Erp et al., 2014; Andon, 2008).

De forma global, esses resultados possuem relevância frente à literatura sobre o tema e aos estudos desenvolvidos anteriormente de três formas: primeiro, provê evidências empíricas da forma indireta pela qual o desempenho de expatriados pode ser influenciado pela adaptação de seus cônjuges. Segundo, traz evidências específicas do contexto brasileiro, como ambiente hospedeiro, o que é particularmente relevante dada a escassez de pesquisas deste tipo em países em desenvolvimento (Araujo, Bilsky, \& Moreira, 2012; Araujo et al., 2014). Terceiro, encontra-se alicerçado em uma amostra expressiva, em comparação a estudos similares. Em conjunto, esses três fatores posicionam esse estudo em uma posição de relevância e originalidade no campo de estudos em Gestão Internacional de Recursos Humanos.

\section{Considerações finais}

Este estudo possui algumas limitações, geradoras de oportunidades de pesquisas futuras. Primeiro, o corte transversal da pesquisa não permite realizar afirmações de natureza causal. Sugere-se, então, que estudos longitudinais sejam realizados de forma que os resultados não sejam apenas interpretados na forma de associações, mas também com relações de causalidade. Segundo, o estudo se restringe a expatriados ao contexto brasileiro, e pode ser que características idiossincráticas desse ambiente tenham gerado vieses para os resultados. Desta forma, propõe-se serem realizados outros estudos com expatriados alocados em outros países para efeito de comparação em outros ambientes hospedeiros. Terceiro, o método de amostragem de bola de neve apresenta como limitação o fato de tender a gerar amostras enviesadas e homogêneas, por se tratarem de grupos de pessoas indicadas por outras, as quais já compunham o banco de participantes da pesquisa. No entanto, o tamanho da amostra obtida parece justificar a escolha pelo método. De qualquer forma, para efeitos de comparação, seria interessante realizar uma replicação do estudo, por exemplo em uma amostra composta por gestores de uma mesma empresa ou de bancos de registros de expatriados para fins de comparação de resultados. Quarto, o estudo não avaliou se a adaptação do cônjuge e do expatriado é suscetível à influência de ações organizacionais como os pacotes de settling in. A fim de superar essa limitação, sugere-se que pesquisas futuras analisem a relação entre a adaptação transcultural dos envolvidos e o suporte organizacional.

Os resultados permitem sugestões para a prática da Gestão Internacional de Recursos Humanos direcionadas a companhias que promovem a expatriação, às empresas fornecedoras de suporte à adaptação de expatriados e suas famílias e aos expatriados em si. Para as empresas, o estudo ressalta a importância de considerar o papel do cônjuge na seleção e gestão da permanência do expatriado durante o período da missão internacional. Entrevistas com a família, prática adotada por algumas organizações, parecem ser necessárias, porquanto é possível que assim como expatriados possuem perfis de valores mais ou menos propícios à adaptação transcultural (Araujo et al., 2012), é razoável sugerir que o mesmo tende a ocorrer com seus cônjuges. Ademais, a geração de incentivos à adaptação do cônjuge parece se tornar uma etapa essencial para o processo, e isso deve incluir desde etapas pré expatriação até o settling in e a repatriação. Para as empresas que fornecem suporte às companhias promovedoras da expatriação, esta pesquisa fornece indícios da relevância de seus serviços e pode ser usada como evidência científica da necessidade de se estender os pacotes de apoio organizacional durante a missão internacional ao cônjuge do expatriado. Por sua vez, o executivo em expatriação pode ganhar maior consciência da necessidade de gerenciar as relações familiares e atentar-se ao suporte emocional demandado por seu cônjuge.

\section{Referências}

- Anderson, N., Ones, D. S., Sinangil, H. K., \& Viswesvaran, C. (Eds.). (2001). Handbook of industrial, work \& organizational psychology: Volume 1: p. 409-423. Personnel psychology. Sage.

- Andreason, A. W. (2008). Expatriate adjustment of spouses and expatriate managers: An integrative research review. International Journal of Management, 25(2), 382-395. 
- Araujo, B. F. V. B., Bilsky, W., \& de Oliveira Moreira, L. M. C. (2012). Valores pessoais como antecedentes da adaptação transcultural de expatriados. Revista de Administração Mackenzie, 13(3), 69-95. http://dx.doi.org/10.1590/S167869712012000300005

- Araujo, B. F. V. B., Teixeira, M. L. M., da Cruz, P. B., \& Malini, E. (2012). Adaptação de expatriados organizacionais e voluntários: similaridades e diferenças no contexto brasileiro. Revista de Administração, 47(4), 555-570. http://dx.doi.org/10.5700/rausp1058

- Araujo, B. F.V. B, Teixeira, M. L. M., da Cruz, P. B., \& Malini, E. (2014). Understanding the adaptation of organisational and self-initiated expatriates in the context of Brazilian culture. The International Journal of Human Resource Management, 25(18), 2489-2509.

http://dx.doi.org/10.1080/09585192.2012.74347 0

- Bhaskar-Shrinivas, P., Harrison, D. A., Shaffer, M. A., \& Luk, D. M. (2005). Input-based and timebased models of international adjustment: Metaanalytic evidence and theoretical extensions. Academy of Management Journal, 48(2), 257-281. http://dx.doi.org/10.5465/AMJ.2005.16928400

- Bauer, T. N., \& Taylor, M. S. (2001). Toward a globalized conceptualization of organizational socialization. International handbook of industrial, work, and organizational psychology, 1, 409-423. http://dx.doi.org/10.4135/9781848608320.n20

- Black, J. S. (1988). Work role transitions: A study of American expatriate managers in Japan. Journal of International Business Studies, 19(2), 277-294.

- Black, J. S., \& Stephens, G. K. (1989). The influence of the spouse on American expatriate adjustment and intent to stay in Pacific Rim overseas assignments. Journal of management, 15(4), 529544.

- Black, J. S., \& Gregersen, H. B. (1991). The other half of the picture: Antecedents of spouse crosscultural adjustment. Journal of international business studies, 22(3), 461-477.

- BGRS. Global relocation trends. (2014) survey report. New York: Windham International, 2014. Recuperado de, em 10 jul 2015: http://www.brookfieldgrs.com/insightsideas/tren ds.asp.

- Brown, R. J. (2008). Dominant stressors on expatriate couples during international assignments. The International Journal of Human Resource Management, 19(6), 1018-1034. http://dx.doi.org/10.1080/09585190802051303

- Caligiuri, P. M. (2000). The big five personality characteristics as predictors of expatriate's desire to terminate the assignment and supervisor-rated performance. Personnel Psychology, 53(1), 67-88. http://dx.doi.org/10.1111/j.17446570.2000.tb00194.x

- Caligiuri, P. M., \& Tung, R. L. (1999). Comparing the success of male and female expatriates from a USbased multinational company. International Journal of Human Resource Management, 10(5), 763-782.

- $\quad$ Cheng, H. L., \& Lin, C. Y. Y. (2009). Do as the large enterprises do?: Expatriate selection and overseas performance in emerging markets: The case of Taiwan SMEs. International Business Review, 18(1), 60-75.

- Chin, W. W. (1998). The partial least squares approach to structural equation modeling. Modern methods for business research, 295(2), 295-336.

- Claus, L., Lungu, A. P., \& Bhattacharjee, S. (2011). The effects of individual, organizational and societal variables on the job performance of expatriate managers. International Journal of Management, 28(1), 249-272.

- Cohen, J. (1997). Statistical power analysis for the behavioral sciences. Revised edition. New York: Academic Press, 1997.

- Cole, N. D. (2011). Managing global talent: Solving the spousal adjustment problem. The International Journal of Human Resource Management, 22(07), 1504-1530. http://dx.doi.org/10.1080/09585192.2011.56196 3

- Collings, D. G., Scullion, H., \& Morley, M. J. (2007). Changing patterns of global staffing in the multinational enterprise: Challenges to the conventional expatriate assignment and emerging alternatives. Journal of World Business, 42(2), 198213. http://dx.doi.org/10.1016/j.jwb.2007.02.005

- Davies, S., Kraeh, A., \& Froese, F. (2015). Burden or support? The influence of partner nationality on expatriate cross-cultural adjustment. Journal of Global Mobility, 3(2), 169-182. http://dx.doi.org/10.1108/JGM-06-2014-0029

- Forster, N., \& Johnsen, M. (1996). Expatriate management policies in UK companies new to the international scene. International Journal of 
Human Resource Management, 7(1), 177-205. http://dx.doi.org/10.1080/09585199600000124

- Hair, J. F., Black, W. C., Babin, B. J., Anderson, R. E., \& Tatham, R. L. (2005). Análise multivariada de dados. Bookman Editora.

- Harvey, M. (1998). Dual-career couples during international relocation: The trailing spouse. International Journal of Human Resource Management, 9(2), 309-331. http://dx.doi.org/10.1080/095851998341116

- Harvey, M., Napier, N., \& Moeller, M. (2009). Interpreting dual career couples' family life-cycles: Identifying strategic windows of global career opportunity. Research and practice in human resource management, 17(2), 14-35.

- Heckathorn, D. D. (1997). Respondent-driven sampling: a new approach to the study of hidden populations. Social problems, 44(2), 174-199.

- Hemmasi, M., Downes, M., \& Varner, I. I. (2010). An empirically-derived multidimensional measure of expatriate success: reconciling the discord. The International Journal of Human Resource Management, 21(7), 982-998. http://dx.doi.org/10.1080/09585191003783447

- Konopaske, R., Robie, C., \& Ivancevich, J. M. (2005). A preliminary model of spouse influence on managerial global assignment willingness. The International Journal of Human Resource Management, 16(3), 405-426. http://dx.doi.org/10.1080/095851904200033957 0

- Kraimer, M. L., Wayne, S. J., \& Jaworski, R. A. A. (2001). Sources of support and expatriate performance: The mediating role of expatriate adjustment. Personnel Psychology, 54(1), 71-99. http://dx.doi.org/10.1111/j.17446570.2001.tb00086.x

- Kraimer, M. L., \& Wayne, S. J. (2004). An examination of perceived organizational support as a multidimensional construct in the context of an expatriate assignment. Journal of Management, 30(2), 209-237. http://dx.doi.org/10.1016/j.jm.2003.01.001

- Kupka, B., \& Cathro, V. (2007). Desperate housewives-social and professional isolation of German expatriated spouses. The International Journal of Human Resource Management, 18(6), 951-968.

http://dx.doi.org/10.1080/09585190701320908

- Kumar, N., Che Rose, R., \& Sri Ramalu, S. (2008). The effects of personality and cultural intelligence on international assignment effectiveness: a review. Journal of Social Sciences, 4(4), 320-328. http://dx.doi.org/10.3844/jssp.2008.320.328

- Lazarova, M. B., \& Cerdin, J. L. (2007). Revisiting repatriation concerns: Organizational support versus career and contextual influences. Journal of International Business Studies, 38(3), 404-429. http://dx.doi.org/10.1057/palgrave.jibs. 8400273

- Lee, Hung-Wen. (2007). Factors that influence expatriate failure: An interview study. International Journal of Management, 24(3), 403-414.

- Lee, L. Y., \& Kartika, N. (2014). The influence of individual, family, and social capital factors on expatriate adjustment and performance: The moderating effect of psychology contract and organizational support. Expert Systems with Applications, 41(11), 5483-5494. http://dx.doi.org/10.1016/j.eswa.2014.02.030

- Lee, L. Y., \& Sukoco, B. M. (2010). The effects of cultural intelligence on expatriate performance: The moderating effects of international experience.The International Journal of Human Resource Management, 21(7), 963-981. http://dx.doi.org/10.1080/0958519100378339

- Lee, L. Y., \& Van Vorst, D. (2010). The influences of social capital and social support on expatriates' cultural adjustment: an empirical validation in Taiwan.International Journal of Management, 27(3), 628-648.

- McNulty, Y., De Cieri, H., \& Hutchings, K. (2009). Do global firms measure expatriate return on investment? An empirical examination of measures, barriers and variables influencing global staffing practices. The International Journal of Human Resource Management, 20(6), 1309-1326. http://dx.doi.org/10.1080/09585190902909830

- Mohr, A. T., \& Klein, S. (2004). Exploring the adjustment of American expatriate spouses in Germany. The International Journal of Human Resource Management, 15(7), 1189-1206. http://dx.doi.org/10.1080/095851904200023840 0

- Pattie, M., \& Parks, L. (2011). Adjustment, turnover, and performance: the deployment of minority expatriates. The International Journal of Human Resource Management, 22(10), 22622280.

http://dx.doi.org/10.1080/09585192.2011.58019 5

- Ramalu, S. S.; Wei, C. C.; Rose, R. C. (2011). The effects of cultural intelligence on cross-cultural adjustment and job performance amongst 
expatriates in Malaysia. International Journal of Business and Social Science, 2(9), 59-72. http://dx.doi.org/10.1080/09585192.2011.59995 6

- Reis, G. G., Fleury, M. T. L., Fleury, A. C. C., \& Zambaldi, F. (2015). Brazilian Multinationals Competences: Impacts of a" Tug of War" Between Cultural Legacies and Global Mindedness. Brazilian Business Review, 12(1), 55-79. http://dx.doi.org/10.15728/bbr.2015.12.1.3

- $\quad$ Ringle, C. M.; Wende, S.; Will, A. (2014). Smart PLS $2.0 \mathrm{M} 3$ (beta). Germany: Universityof Hamburg, 2005. Recuperado de, em 22 ago. 2014: <http://www.smartpls.de>.

- Santos, N. M., Bronzo, M., Oliveira, M. P. V., \& Resende, P. T. V. (2014). Organizational Culture, Organizational Structure and Human Resource Management as Bases for Business Process Orientation and their Impacts on Organizational Performance. Brazilian Business Review, 11(3), 100-122. http://dx.doi.org/10.15728/bbr.2014.11.3.5

- Schuler, R. S., Fulkerson, J. R., \& Dowling, P. J. (1991). Strategic performance measurement and management in multinational corporations. Human Resource Management, 30(3), 365-392. http://dx.doi.org/10.1002/hrm.3930300305

- Selmer, J., \& Leung, A. S. (2003). International adjustment of female vs male business expatriates. International Journal of Human Resource Management,14(7), 1117-1131. http://dx.doi.org/10.1108/JGM-12-2014-0055

- Selmer, J., \& Fenner Jr, C. R. (2009). Spillover effects between work and non-work adjustment among public sector expatriates. Personnel Review, 38(4), 366-379. http://dx.doi.org/10.1108/00483480910956328

- Shaffer, M. A., \& Harrison, D. A. (1998). Expatriates' psychological withdrawal from international assignments: work, nonwork, and family influences. Personnel psychology, 51(1), 87 118. http://dx.doi.org/10.1111/j.17446570.1998.tb00717.x

- Shaffer, M. A., \& Harrison, D. A. (2001). Forgotten partners of international assignments: development and test of a model of spouse adjustment. Journal of Applied Psychology, 86(2), 238-254. http://dx.doi.org/10.1037/0021-
- Shaffer, M. A., Harrison, D. A., Gregersen, H., Black, J. S., \& Ferzandi, L. A. (2006). You can take it with you: individual differences and expatriate effectiveness. Journal of Applied psychology, 91(1), 109-125. http://dx.doi.org/10.1037/0021-9010.91.1.109

- Shih, H. A., Chiang, Y. H., \& Hsu, C. C. (2010). High involvement work system, work-family conflict, and expatriate performance-examining Taiwanese expatriates in China. The International Journal of Human Resource Management, 21(11), 2013-2030.

http://dx.doi.org/10.1080/09585192.2010.50510 1

- Takeuchi, R., Yun, S., \& Tesluk, P. E. (2002). An examination of crossover and spillover effects of spousal and expatriate cross-cultural adjustment on expatriate outcomes. Journal of applied psychology, 87(4), 655-666. http://dx.doi.org/10.1037//0021-9010.87.4.655

- $\quad$ Takeuchi, R., Lepak, D. P., Marinova, S. V., \& Yun, S. (2007). Nonlinear influences of stressors on general adjustment: the case of Japanese expatriates and their spouses. Journal of International Business Studies, 38(6), 928-943. http://dx.doi.org/10.1057/palgrave.jibs.8400298

- Van der Zee, K. I., Ali, A. J., \& Salomé, E. (2005). Role interference and subjective well-being among expatriate families. European Journal of Work and Organizational Psychology, 14(3), 239-262. http://dx.doi.org/10.1080/13594320500146250

- Van Erp, K. J., van der Zee, K. I., Giebels, E., \& van Duijn, M. A. (2014). Lean on me: The importance of one's own and partner's intercultural personality for expatriate's and expatriate spouse's successful adjustment abroad. European journal of work and organizational psychology, 23(5), 706-728. http://dx.doi.org/10.1080/1359432X.2013.81608 8

- Wu, P. C., \& Ang, S. H. (2011). The impact of expatriate supporting practices and cultural intelligence on cross-cultural adjustment and performance of expatriates in Singapore. The International Journal of Human Resource Management, 22(13), 2683-2702. http://dx.doi.org/10.1080/09585192.2011.59995 6

9010.86 .2 .238 


\title{
SOBRE OS AUTORES
}

- Brunella Tristão Simonelli é Mestre em Administração pela Fundação Instituto Capixaba de Pesquisas em Contabilidade, Economia e Finanças - FUCAPE. Proprietária da Talento - Assessoria e Gestão em Recursos Humanos, dede 2003. É coordenadora do MBA em Gestão de Pessoas e docente da disciplina de psicologia e administração da Faculdade Pitágoras. E-mail: brunella@talentorh.net.

- Bruno Felix von Borell de Araujo é Professor nos cursos de graduação e pós-graduação lato e stricto sensu na Fucape Business School. Coordenador dos MBAs em Gestão Estratégica de Pessoas e Liderança e Desenvolvimento Humano e de MBAs In Company na mesma instituição. E-mail: bfelix@fucape.br.ORCID: http//:0000-0001-6183-009X

\section{The Adjustment of Spouses and Expatriates and its relationship with expatriate's performance in international assignments}

\author{
Brunella Tristão Simonelli ${ }^{A}$ e Bruno Felix von Borell de Araujo ${ }^{B}$ \\ ${ }^{A}$ Faculty Pythagoras, Linhares, ES, Brazil \\ ${ }^{B}$ Capixaba Fundation Institute of Research in Accounting, Economics and Finance - FUCAPE, Vitória, ES, Brazil
}

\section{ARTICLE DETAILS}

\section{Article history:}

Received 22 July 2015

Accepted 24 March 2016

Available online in 31 April 2016

Double Blind Review System

Scientific Editor

Eduardo Eugênio Spers

\section{Keywords:}

Adaptation

Expatriate

Spouse

Performance

\begin{abstract}
The adaptation of an expatriate is a function of several factors and family issues have been identified as potential influencers in the process. However, little is known about the effect of the existing role of the spouse in the adaptation of the expatriate. Thus, the objective of this study is to evaluate the relationship between the adjustment of expatriates and their spouses with the performance of the expatriate. It is a quantitative study with 217 couples in which one member of the couple occupied the expatriate position. The results showed no significant relationship between the adjustment of the spouse and the performance of the expatriate, but revealed that the adaptation of expatriate moderates the relationship between these variables. These results suggest that the adaptation of the spouse can have an indirect relationship on expatriate performance, via the expatriate's adaptation. These findings support the idea that companies should invest in organizational support to facilitate a better fit of the expatriate and his spouse to the host country culture and to promote a higher individual performance.
\end{abstract}

(C) 2016 Internext | ESPM. Todos os direitos reservados!

Para citar este artigo:

Simonelli, B. T. \& Araujo, B. F. V. B. (2016) Adaptação de Cônjuges e de Expatriados e sua relação com o Desempenho em Designações Internacionais. Internext - Revista Eletrônica de Negócios Internacionais, 11 (2), p. 21-34.

Para acessar este artigo: $h t t p: / / d x . d o i . o r g / 10.18568 / 1980-4865.11221-34$ 